\title{
Refractory Fallopian Tube Carcinoma
}

National Cancer Institute

\section{Source}

National Cancer Institute. Refractory Fallopian Tube Carcinoma. NCI Thesaurus. Code C152047.

Fallopian tube carcinoma that is resistant to treatment. 